\title{
Tactile spatial acuity differs between fingers: A study comparing two testing paradigms
}

\author{
ARTHUR C. GRANT \\ New York University School of Medicine \\ and \\ RAQUEL FERNANDEZ, PARASTOU SHILIAN, ELIZABETH YANNI, and MARY ANN HILL \\ University of California, Irvine, California
}

\begin{abstract}
Tactile spatial acuity (TSA) is a reliable and reproducible measure of somatosensory system function that has been used to study a broad range of subject populations. Although TSA is most often assessed at the fingertip, published studies employing identical stimuli disagree on whether TSA differs between the fingers of neurologically normal subjects. Using a validated grating orientation discrimination task, we determined TSA bilaterally at the index and ring fingers of 16 healthy young adults. Motivated by earlier work, we utilized two stimulus presentation paradigms, the method of constant stimuli (MCS) and a staircase (SC) method. We found that TSA was significantly higher (the discrimination threshold was lower) at the index than at the ring finger, which was consistent with a prior study. Although mean thresholds at both fingers were higher when measured with the SC than with the MCS paradigm, this difference did not reach statistical significance $(p=.14)$. These findings should facilitate both design and interpretation of future studies investigating TSA.
\end{abstract}

Tactile spatial acuity (TSA) is a reliable indicator of somatosensory system integrity, and it is highly correlated with subjective tactile perceptual dysfunction (Johnson \& Hsiao, 1992; Johnson \& Phillips, 1981; Van Boven \& Johnson, 1994a, 1994b). A well-established and reproducible measure of TSA is the psychophysical threshold for discrimination of grating orientation (Johnson \& Phillips, 1981; Sathian \& Zangaladze, 1996; Van Boven \& Johnson, 1994a, 1994b). To obtain this measure, gratings varying in spatial frequency are applied to a body part and the subject states the stimulus orientation with respect to a bodycentered axis, in a forced choice paradigm, resulting in a grating orientation discrimination threshold (GOT). This measure of TSA has been applied to the study of peripheral nerve injury (Van Boven \& Johnson, 1994b), Braille readers (Goldreich \& Kanics, 2003; Grant, Thiagarajah, \& Sathian, 2000; Van Boven, Hamilton, Kauffman, Keenan, \& Pascual-Leone, 2000), Parkinson's disease (Sathian, Zangaladze, Green, Vitek, \& DeLong, 1997), dyslexia (Grant, Zangaladze, Thiagarajah, \& Sathian, 1999), and age-related changes in spatial acuity (Sathian et al., 1997; Tremblay, Backman, Cuenco, Vant, \& Wassef, 2000; Vega-Bermudez \& Johnson, 2004), as well as in the study of other subject populations. Surprisingly, despite the fre-

This study was supported in part by K23 NS46347, UCI Health Science Partners Research Award, and UCI College of Medicine Committee on Research, all to A.C.G. Correspondence should be addressed to A. C. Grant, NYU Comprehensive Epilepsy Center, 403 East 34th St., 4th Floor, New York, NY 10016 (e-mail: arthur.grant@med.nyu.edu). quent application of this measure at the human fingertip, there is no consensus on whether TSA differs between the fingers of neurologically normal subjects. Furthermore, different stimulus presentation paradigms may produce different GOT values.

Two studies have reported normative GOT values at the different fingers. One study (Sathian \& Zangaladze, 1996; $N=7$ subjects; mean age, 27.6 years) found equivalent thresholds at the first through the fourth digit, whereas another study (Vega-Bermudez \& Johnson, 2001; 8 subjects; mean age, 34.8 years) found that the GOT increased significantly from the second to the third digit, and from the third to the fourth digit. Studies comparing the GOT at the index fingertip between a variety of subject populations and nonelderly normal controls ( $<55$ years) have reported mean normal values ranging from $0.82 \mathrm{~mm}$ (Sathian \& Zangaladze, 1997) up to $1.57 \mathrm{~mm}$ (Van Boven et al., 2000; see their Figure 2). In the latter study, a GOT of $0.97 \mathrm{~mm}$ in blind Braille readers was interpreted to represent supranormal performance (Van Boven et al., 2000).

Vega-Bermudez and Johnson (2004) have recently shown that in young subjects, natural variation in fingertip skin conformance accounts for about $50 \%$ of the statistical variation in the tactile GOT. Another factor that may account for some between-studies variation in normal values is the testing method. The two stimulus presentation paradigms utilized in these studies, the method of constant stimuli (MCS) and the staircase (SC) method, both result in a quantitative psychophysical threshold. Nonetheless, there are potentially significant qualitative differences. For instance, with the SC method, the subject is never ex- 
posed to a single grating consecutively for more than two trials, whereas in the MCS, each grating is applied in a block of 20-50 consecutive trials. Such repeated and expected experience with a single grating may improve overall performance. Furthermore, with the SC method, the subject experiences stimuli near threshold (e.g., 1.2-mm grating) after as few as 6 trials, whereas with the MCS, this may require 60-150 trials.

Until there is agreement on whether TSA differs between fingers and whether the psychophysical tactile GOT is independent of testing paradigm, comparing data between both subject and research groups will be unnecessarily difficult. The present study was undertaken to answer these questions, by using a large number of subjects to increase statistical power, and by directly comparing the MCS and the SC method.

\section{METHOD}

Subjects consisted of 16 right-handed adults (mean age, 23.5 years) with no history of learning disability, fingertip calluses, or neurological disease including peripheral nerve injury or dysfunction. Although age does not seem to affect the psychophysical threshold for grating orientation at the fingertip until at least the sixth decade (Sathian et al., 1997; Tremblay et al., 2000; Vega-Bermudez \& Johnson, 2004), we intentionally used a relatively young adult population to avoid age as a possible confounding factor. The study was approved by the University of California, Irvine Institutional Review Board, and each subject provided written informed consent.

Tactile grating orientation discrimination was determined at the index (D2) and ring (D4) fingers of both hands using square-wave gratings cut into the face of plastic domes (JVP Domes, Stoelting, Wood Dale, IL), with equal ridge and groove widths $(0.35,0.5,0.75,1.0$, $1.2,1.5,2.0,3.0 \mathrm{~mm})$. The subjects were seated comfortably in a quiet room and were blindfolded during the testing. The finger being tested was immobilized with double-sided tape applied to its dorsal surface. In each trial, the grating was applied for about $1 \mathrm{sec}$ with moderate force normal to the distal fingerpad. The grating was aligned parallel or perpendicular to the long axis of the finger, and the subject had to state the grating orientation in a two-alternative forced choice paradigm.

To vary testing order, subjects were assigned in a pseudorandom fashion into four groups of 4 subjects each. Testing was equally likely to begin with any of the four fingers and with either the MCS or SC paradigm. Finger order and testing paradigm were then fixed so that testing alternated between hands, and one paradigm was used on one hand while the other paradigm was used on the other hand. Since TSA at the fingertip of normal subjects is symmetric (Sathian \& Zangaladze, 1996; Vega-Bermudez \& Johnson, 2001), a consistent within-subjects GOT asymmetry would indicate differential subject performance with the MCS and SC methods.

The use of the MCS paradigm was based on previous reports (Grant et al., 2000; Grant et al., 1999; Sathian \& Zangaladze, 1996; Sathian et al., 1997; Van Boven \& Johnson, 1994a). Briefly, each grating was applied in a block of 50 trials containing an equal number of parallel and perpendicular applications in a pseudorandom sequence. Testing began with either the $3.0-$ or the $2.0-\mathrm{mm}$ grating and continued until performance fell below the psychophysical threshold of $71 \%$ correct on two successive gratings. A threshold of $71 \%$ was used rather than the standard $75 \%$ because the SC protocol converges on a threshold of $71 \%$ correct, but both values were calculated to facilitate comparison with other studies. The thresholds were calculated by linear interpolation between the scores for the gratings just above and just below threshold performance.

The use of the SC method was also based on previous reports (Vega-Bermudez \& Johnson, 2001; Wetherill \& Levitt, 1965). This method generally requires many fewer trials than the MCS, which is particularly useful when several thresholds must be determined in one testing session. The SC method converges on a threshold corresponding to any one of several fixed percent correct responses. As used here, it converges on $70.7 \%$. Testing began with two trials of the $3.0-\mathrm{mm}$ grating. If the subject answered both trials correctly, then the next most difficult grating was applied. An incorrect answer for the first or second trial of a grating resulted in switching to the next easier grating, whereas correct answers for two successive trials resulted in switching to the next harder grating. Testing continued for 16 reversals, and the grating spatial frequency corresponding to the final 12 reversals was averaged to arrive at the threshold.

\section{Statistical Analysis}

Threshold data were analyzed using a repeated measures ANOVA with finger (D2 and D4) and test paradigm (MCS and SC) as withinsubjects factors, subject group as a between-subjects factor, and GOT as the dependent variable. Possible learning effects during the MCS testing session were also investigated. A paired $t$ test was used to compare, for each subject, the total number of incorrect responses during the first 25 trials with that during the last 25 trials, for all gratings used on the first finger tested with the MCS paradigm.

\section{RESULTS}

\section{GOT at D2 and D4}

Thresholds for individual subjects are shown in Table 1. There was a significant within-subjects effect of finger tested (D2 vs. D4, $F=37.7, p<.0005$ ). Given only the MCS data and the standard psychophysical threshold of $75 \%$ correct, the mean GOT was 1.12 at D2 and $1.65 \mathrm{~mm}$ at D4.

\section{Comparison of MCS and SC Method}

The mean GOT was higher with the SC method than with the MCS for both digits (1.21 vs. $1.06 \mathrm{~mm}$ at D2 and 1.60 vs. $1.51 \mathrm{~mm}$ at D4), although these differences did not reach statistical significance $(F=2.5, p=.14)$. There was no effect of subject group (i.e., there was no order effect; $F=0.7, p=.59$ ), and there was no interaction between finger tested and testing paradigm $(F=0.2, p=$ .66). Also, there was no interaction of finger or testing paradigm with subject group.

\section{Learning During MCS Testing}

There was no within-subjects difference between total number of incorrect responses during the first and last 25 trials with the MCS paradigm $(p=.46)$.

\section{DISCUSSION}

\section{TSA at D2 and D4}

Our data confirm that TSA is higher (the GOT is lower) at the index than at the ring finger, and that TSA at the index fingertip of most normal young adults falls between 0.90 and $1.20 \mathrm{~mm}$. We did not measure GOT at the other digits, but given the quantitative similarity of our results at D2 and D4 to those of a prior study (Vega-Bermudez \& Johnson, 2001), it is reasonable to conclude that the TSA of D3 falls between that of D2 and D4, as reported in the earlier study (Vega-Bermudez \& Johnson, 2001). These normative data and the associated demonstration of a defi- 
Table 1

Grating Orientation Discrimination Thresholds at the Second (D2) and Fourth (D4) Digit Using the Method of Constant Stimuli (MCS) or Staircase (SC) Paradigm

\begin{tabular}{cccccccc}
\hline & & & & \multicolumn{4}{c}{$\begin{array}{c}\text { Grating Orientation } \\
\text { Discrimination Threshold (mm) }\end{array}$} \\
\cline { 5 - 7 } Group & Subject & $\begin{array}{c}\text { Age } \\
\text { (Years) }\end{array}$ & Sex & MCS D2 & SC D2 & MCS D4 & SC D4 \\
\hline 1 & 1 & 19 & M & 1.08 & 1.12 & 1.49 & 1.99 \\
1 & 2 & 27 & M & 1.34 & 0.92 & 1.48 & 1.13 \\
1 & 3 & 26 & M & 0.97 & 0.98 & 1.36 & 1.44 \\
1 & 4 & 29 & F & 0.99 & 0.78 & 1.64 & 1.39 \\
2 & 5 & 19 & F & 0.93 & 1.08 & 1.43 & 1.14 \\
2 & 6 & 24 & M & 0.89 & 0.88 & 1.03 & 1.84 \\
2 & 7 & 23 & M & 1.13 & 1.08 & 1.45 & 1.39 \\
2 & 8 & 27 & F & 1.01 & 1.08 & 1.54 & 1.56 \\
3 & 9 & 18 & F & 1.04 & 1.78 & 1.14 & 1.86 \\
3 & 10 & 22 & F & 1.13 & 0.86 & 1.71 & 1.88 \\
3 & 11 & 30 & F & 1.15 & 2.35 & 2.63 & 2.31 \\
3 & 12 & 24 & M & 0.89 & 1.02 & 1.35 & 1.52 \\
4 & 13 & 19 & F & 1.14 & 1.66 & 1.39 & 2.03 \\
4 & 14 & 22 & M & 0.76 & 0.82 & 0.94 & 0.96 \\
4 & 15 & 19 & F & 1.10 & 0.90 & 0.96 & 0.79 \\
4 & 16 & 28 & M & 1.37 & 2.07 & 2.65 & 2.31 \\
Mean & & 23.5 & & 1.06 & 1.21 & 1.51 & 1.60 \\
SE & & 1.0 & & .04 & .12 & .12 & .11 \\
\hline Note- Subject number reflects an arbitrary posttest assignment. Individual subjects
\end{tabular}
were assigned to test groups as described in the text.

nite difference in TSA between D2 and D4 should assist future studies of both neurologically normal subjects and those with hypothesized impaired or supranormal tactile function at the fingertip.

The mean D2 GOT of $1.12 \mathrm{~mm}$, obtained with the MCS paradigm and the conventional psychophysical threshold of $75 \%$ correct, is quite similar to values reported in several prior studies (Grant et al., 2000; Grant et al., 1999; Grant, Henry, Fernandez, Hill, \& Sathian, 2005; Van Boven \& Johnson, 1994a; Vega-Bermudez \& Johnson, 2004), whereas the mean D2 threshold of $1.21 \mathrm{~mm}$ using the SC method is nearly identical to that reported in the only prior study using this paradigm to study normal subjects (Vega-Bermudez \& Johnson, 2001). At D4, our mean threshold of $1.60 \mathrm{~mm}$ with the $\mathrm{SC}$ method is also very close to the threshold of $1.64 \mathrm{~mm}$ reported in the prior study that used this method and that found a significant difference between thresholds at D2 and D4 (Vega-Bermudez \& Johnson, 2001). The mean D4 GOT of $1.65 \mathrm{~mm}$ (MCS, psychophysical threshold of $75 \%$ correct) is substantially higher than the $1.06 \mathrm{~mm}$ reported in the study using this paradigm that found no TSA difference between D2 and D4 (Sathian \& Zangaladze, 1996). Methodological limitations of that study have been described (Vega-Bermudez \& Johnson, 2001), and they include a relatively small number of subjects, a small block size of 20 trials, and the combining of data from different testing sessions to arrive at an overall threshold for each finger.

The variability of TSA with anatomical location may be due to corresponding differences in mechanoreceptive fiber density and/or cortical magnification factor (Sathian \& Zangaladze, 1996; Van Boven et al., 2000; Van Boven \& Johnson, 1994a, 1994b; Vega-Bermudez \& Johnson,
2001), either of which may account for the difference in TSA at the index and ring fingers. The relative density of the pertinent fiber type - slowly adapting (Merkel) afferents (Phillips \& Johnson, 1981) - at the human fingertips is unknown. In addition, the relationship between TSA and cortical magnification factor of the corresponding body part is not straightforward. In a study of 15 patients with amputated digits and an equal number of controls, Vega-Bermudez and Johnson (2002) demonstrated that TSA was not increased at the digit adjacent to the amputation in comparison with the same digit on the contralateral hand, nor was it increased in comparison with control subjects. Thus, the increased cortical representation of digits adjacent to an amputation (first shown by Merzenich et al. [1984] in monkeys) does not necessarily result in improved function, at least to the extent that TSA reflects somatosensory abilities. The complexity of this relationship clearly deserves additional investigation.

\section{Comparison of the MCS and the SC Method}

Prior experience with this task (Grant et al., 2005; Grant et al., 2000; Grant et al., 1999) led us to hypothesize that the considerably greater exposure to test stimuli in the MCS paradigm would result in optimal performance, reflecting the subject's genuine spatial acuity. Indeed, mean GOT at both D2 and D4 was lower with the MCS than with the SC method, but, contrary to our expectations, these differences were relatively small and did not reach statistical significance. Although a study with greater statistical power could theoretically demonstrate a true difference in subject performance with these methods, the relatively large number of subjects with a limited age distribution, coupled with a conservative statistical analysis, leads us 
to conclude that both methods produce a meaningful measure of TSA. In fact, one could argue that the increased duration of testing and number of stimulus applications in the MCS would increase likelihood of fatigue, inattentiveness, and a subjective feeling of numbness at the stimulus site, each of which could adversely affect performance. That practice effects within an MCS test session were not significant is supported by the absence of a withinsubjects performance difference between the first and last 25 trials with each grating used during a complete MCS session. Thus, the method chosen to administer this task, tactile discrimination of grating orientation, should depend on experiment-specific factors, such as the number of subjects, sites to be tested, testing sessions, and whether any other procedures will be performed.

\section{REFERENCES}

GoldREICH, D., \& KanICS, I. M. (2003). Tactile acuity is enhanced in blindness. Journal of Neuroscience, 23, 3439-3445.

Grant, A. C., Henry, T. R., Fernandez, R., Hill, M. A., \& Sathian, K. (2005). Somatosensory processing is impaired in temporal lobe epilepsy. Epilepsia, 46, 534-539.

Grant, A. C., Thiagarajah, M. C., \& Sathian, K. (2000). Tactile perception in blind Braille readers: A psychophysical study of acuity and hyperacuity using gratings and dot patterns. Perception \& Psychophysics, 62, 301-312.

Grant, A. C., Zangaladze, A., Thiagarajah, M., \& Sathian, K. (1999). Tactile perception in developmental dyslexia: A psychophysical study using gratings. Neuropsychologia, 37, 1201-1211.

Johnson, K. O., \& HsiaO, S. S. (1992). Neural mechanisms of tactual form and texture perception. Annual Review of Neuroscience, 15, 227 250 .

Johnson, K. O., \& Phillips, J. R. (1981). Tactile spatial resolution: I. Two-point discrimination, gap detection, grating resolution, and letter recognition. Journal of Neurophysiology, 46, 1177-1192.

Merzenich, M. M., Nelson, R. J., Stryker, M. P., Cynader, M. S., Schoppmann, A., \& ZooK, J. M. (1984). Somatosensory cortical map changes following digit amputation in adult monkeys. Journal of Comparative Neurology, 224, 591-605.

Phillips, J. R., \& Johnson, K. O. (1981). Tactile spatial resolution: II. Neural representation of bars, edges, and gratings in monkey primary afferents. Journal of Neurophysiology, 46, 1192-1203.

Sathian, K., \& Zangaladze, A. (1996). Tactile spatial acuity at the human fingertip and lip: Bilateral symmetry and interdigit variability. Neurology, 46, 1464-1466.

Sathian, K., \& Zangaladze, A. (1997). Tactile learning is task specific but transfers between fingers. Perception \& Psychophysics, 59, 119-128.

Sathian, K., Zangaladze, A., Green, J., Vitek, J. L., \& DeLong, M. R. (1997). Tactile spatial acuity and roughness discrimination: Impairment due to aging and Parkinson's disease. Neurology, 49, 168-177.

Tremblay, F., Backman, A., Cuenco, A., Vant, K., \& Wassef, M.-A. (2000). Assessment of spatial acuity at the fingertip with grating (JVP) domes: Validity for use in an elderly population. Somatosensory \& Motor Research, 17, 61-66.

Van Boven, R. W., Hamilton, R. H., Kauffman, T., Keenan, J. P., \& Pascual-Leone, A. (2000). Tactile spatial resolution in blind Braille readers. Neurology, 54, 2230-2236.

VAN Boven, R. W., \& Johnson, K. O. (1994a). The limit of tactile spatial resolution in humans: Grating orientation discrimination at the lip, tongue, and finger. Neurology, 44, 2361-2366.

VAN Boven, R. W., \& Johnson, K. O. (1994b). A psychophysical study of the mechanisms of sensory recovery following nerve injury in humans. Brain, 117, 149-167.

Vega-Bermudez, F., \& Johnson, K. O. (2001). Differences in spatial acuity between digits. Neurology, 56, 1389-1391.

Vega-Bermudez, F., \& Johnson, K. O. (2002). Spatial acuity after digit amputation. Brain, 125, 1256-1264.

Vega-Bermudez, F., \& Johnson, K. O. (2004). Fingertip skin conformance accounts, in part, for differences in tactile spatial acuity in young subjects, but not for the decline in spatial acuity with aging. Perception \& Psychophysics, 66, 60-67.

Wetherill, G. B., \& LeVITt, H. (1965). Sequential estimation of points on a psychometric function. British Journal of Mathematical \& Statistical Psychology, 18, 1-10.

(Manuscript received July 27, 2005; revision accepted for publication January 31,2006 .) 